\title{
Editorial
}

Burnell R. Brown Jr MD PhD FFARCS, Edward J. Frink Jr MD

\section{Whatever happened to sevoflurane?}

quieting fact emerged concerning sevoflurane. Under somewhat severe conditions, sevoflurane would break down in the presence of soda-lime and baralime. Because isoflurane was devoid of both these disturbing aspects, it seemed quite logical at the time to shelve sevoflurane in favour of developing isoflurane. Sevoflurane remained dormant for years.

Eventually a Japanese pharmaceutical company (Maruishi Co., Osaka) questioned some of the condemning data regarding this drug. Their analysis of sevoflurane reactivity in soda-lime demonstrated that considerable breakdown occurred only above $65^{\circ} \mathrm{C}$, temperature levels which are far greater than occur in real-life situations. Although five breakdown products were found during severe experimental conditions, only one of these reaches measurable levels in the anaesthesia circuit. Although not realized by most practicing anaesthetists, halothane has similar high temperature soda-lime catalyzed breakdown. Paradoxically, the breakdown products of halothane under these circumstances are over four times more toxic than the primary breakdown product of sevoflurane! Recent studies of prolonged low-flow anaesthesia $(<750 \mathrm{ml}$ $\mathrm{min}^{-1}$ ) in man by us have shown that only $15 \mathrm{ppm}$ of the major breakdown product of sevoflurane is achieved in anaesthetic circuits, a level hundreds of orders of magnitude lower than toxic levels. What about the problem of biotransformation? Sevoflurane is metabolized to about the same percentage as enflurane $(\sim 3 \%)$. The principal products are hexafluoroisopropanol (HFIP) and inorganic fluoride ion. Hexafluoroisopropanol is rapidly conjugated to the non-toxic glucuronide. In fact, HFIP is so rapidly glucuronidated to inert by-products that covalent bonding of sevoflurane to hepatic macromolecules is theoretically almost impossible. The implication of this is that hepatotoxicity predicated on metaboliteprotein complexes to form haptens is remote. During clinical anaesthesia of several hours duration plasma-free fluoride levels rise to $25-30 \mu \mathrm{M} \cdot \mathrm{L}^{-1}$ similar to that of enflurane. However, here the similarity to enflurane disappears. Enflurane is far more soluble in both lipids and water than is sevoflurane, retention of enflurane molecules in the body is longer and total fluoride accumulation is 
greater than with sevoflurane. Since renal toxicity has not been reported with enflurane, which usually produces more total fluoride than sevoflurane, the fluoride problem with sevoflurane is probably more theoretical than real. Over 250,000 sevoflurane anaesthetics have been administered in Japan since the drug was released in that country in May 1990 and no case of renal damage in the postoperative period has been attributed to the drug. The problem is not entirely resolved because a very few patients $(<10)$ have been reported with blood fluoride levels exceeding $50 \mu \mathrm{M} \cdot \mathrm{L}^{-1}$. None of these patients developed renal problems, probably because of rapid decreases in the fluoride plasma levels. But this issue still remains as the major unknown of sevoflurane.

Sevoflurane is perhaps the least irritant on the respiratory tract of the halogenated anaesthetics. This feature coupled with a very rapid induction (blood/gas partition coefficient $=0.58$ ) makes the drug a paediatric anaesthetic par excellence. One of the authors of the article under editorial consideration (Dr. Lerman) has recently completed the first series of paediatric inhalational inductions with sevoflurane in the Western hemisphere in Toronto. His experience amplifies that of Japanese investigators sevoflurane allows rapid, pleasant, irritation-free inhalational induction in infants and children. Other seminal features of sevoflurane include lack of cardiac "sensitization" to catecholamines, rapid recovery, minimal blood pressure changes and apparently less reflex tachycardia than is seen with available anaesthetics. Many studies similar to that of Crawford et al. attest to minimal disruption of hepatic, coronary, and cerebral blood flow when sevoflurane anaesthesia is employed.

Sevoflurane is certainly not a panacea but it has several desirable characteristics not available with older halogenated inhalation anaesthetics. The problem of biotransformation is an important one and must be studied further, but it may be of little consequence particularly compared to safety obtained in other areas. Sevoflurane seems to have a future as a paediatric "niche" drug. Because it possesses no chlorine atom, it is probably more "ozone friendly" than currently available halogenated anaesthetics. The eventual role of this new/old anaesthetic in our practice is a question for the future.

\section{Oú est donc passé le sévoflurane?}

Crawford et ses collaborateurs signent dans ce numéro du Journal, un article dont le titre est le suivant : « Haemodynamic and organ blood flow responses to sevoflurane during spontaneous ventilation in the rat: a dose response study. " Ils y soulignent que dans les limites de leur modèle, le sévoflurane n'affecte ni la stabilité cardio-vasculaire ni les flux sanguins régionaux. Ces résultats confirment ceux d'autres chercheurs et permettent d'affirmer que chez les animaux, à concentrations équivalentes, le sévoflurane entraîne moins d'effets cardio-vasculaires que les autres anesthésiques en inhalation. Je vous sens perplexes : «Si le sévoflurane est si bon, pourquoi ne pas l'avoir dit plus tôt ?»

Le sévoflurane traîne dans le paysage depuis plus de quinze ans alors que Wallin et ses collaborateurs chez Baxter-Travenol en faisaient la synthèse.' Holaday et Smith $^{2}$ en coordonnèrent les premiers essais sur des volontaires (Phase I) il y a plus de dix ans. Ces premiers essais furent tout à fait satisfaisants et le sévoflurane les passa avec brio. Les raisons alléguées pour expliquer son retard à s'affirmer sur la scène clinique sont complexes. D'abord, on a commencé à s'intéresser à ce nouvel agent au moment où l'isoflurane entamait le dernier droit de sa phase de développement. A prime abord, on peut noter quelques uns des atouts dont disposait l'isoflurane face au sévoflurane, ce nouveau rival potentiel sur la scène clinique et commerciale. Seul $1 \%$ de l'isoflurane absorbé est transformé alors qu'à peu près $3 \%$ du sévoflurane subit le même sort. Puisqu'on visait avec l'isoflurane le marché de l'enflurane (au métabolisme comparable à celui du sévoflurane), il fallait l'en démarquer en insistant sur sa faible biotransformation. Il faut se rappeler qu'à l'époque, les métabolites d'anesthésiques halogénés avaient mauvaise réputation : ceux du méthoxyflurane avaient été incriminés pour leur toxicité rénale et on suspectait ceux de l'halothane d'être hépatotoxiques. Si ce n'était pas assez, on rapporta qu'en certaines circonstances défavorables, le sévoflurane devenait instable en présence de chaux sodée. Comme l'isoflurane n'entraînait pas ce genre d'inquiétude, on s'estima justifié d'en promouvoir le développement quitte à laisser le sévoflurane sur une voie d'évitement où il est longtemps resté.

Survint un jour une petite compagnie pharmaceutique japonaise (Cie Maruishi d'Osaka) qui remit en question la sévérité du jugement imposé au sévoflurane. Son 
travail démontra que l'instabilité du sévoflurane en présence de chaux sodée ne devenait importante que lorsque la température dépassait 65 degrés, soit bien au delà des conditions réelles d'utilisation. Par ailleurs, si on avait au départ identifié cinq dérivés différents à haute température, un seul atteignait un niveau mesurable dans le circuit anesthésique. Même si bien des anesthésistes l'ignorent, l'halothane aussi se dégrade en présence de chaux sodée à haute température et ses dérivés sont au moins quatre fois plus toxiques que ceux du sévoflurane. Nous avons récemment démontré que chez l'homme, une anesthésie prolongée à bas débit (<750 $\left.\mathrm{ml} \cdot \mathrm{min}^{-1}\right)$ n'entraîne l'accumulation que de 15 ppm de dérivés du sévoflurane dans le circuit anesthésique, niveau à des années-lumières du seuil toxique. Qu'en est-il de sa biotransformation ? L'importance du métabolisme du sévoflurane est comparable à celle de l'enflurane soit à peu prés $3 \%$. L'hexafluoroisopropanol (HFIP) et l'ion fluorure en sont les principaux métabolites et l'HFIP est rapidement conjugué au glucuronide, formant un composé inoffensif. Cette réaction est tellement rapide qu'un liaison covalente entre sévoflurane et macromolécules hépatiques est virtuellement impossible. Cela rend fort improbable toute hépatotoxicité à base de complexes métabolite-protéine jouant aux haptènes. Par ailleurs, après une anesthésie de plusieurs heures au sévoflurane, le fluorure inorganique atteint une concentration plasmatique de $25-30 \mu \mathrm{M} \cdot \mathrm{L}^{-1}$ semblable au niveau atteint avec l'enflurane. Toutefois, l'enflurane est beaucoup plus soluble dans l'eau et les graisses que le sévoflurane et ses molécules séjournent plus longtemps in vivo, entraînant une accumulation de fluorure supérieure à celle du sévoflurane. Comme on a pas identifié de cas de toxicité rénale avec l'enflurane, la néphrotoxicité du sévoflurane devrait demeurer à fortiori un sujet théorique. D'ailleurs, on a utilisé ce dernier lors de plus de 250000 interventions au Japon depuis mai 1990 sans pouvoir l'incriminer dans aucun cas de défaillance rénale postopératoire. On a toutefois identifié quelques patients $(<10)$ chez qui le taux de fluorure sanguin dépassa $50 \mu \mathrm{M} \cdot \mathrm{L}^{-1}$ mais aucun d'entre-eux n'eut de problème rénal, probablement grâce à la diminution rapide de ces niveaux. Admettons que tout n'a pas encore été dit quant à cet aspect du sévoflurane.

Comparé aux autres anesthésiques halogénés, le sévoflurane est probablement moins irritant pour les voies respiratoires, avec en prime un coefficient sang/gaz de 0,58 permettant une induction rapide, ce qui en fait un agent de choix en anesthésie pédiatrique. Jerry Lerman, un des auteurs de l'article qui appelle notre commentaire, vient d'ailleurs de faire les premières inductions par inhalation avec le sévoflurane en Occident, à Toronto. A l'instar des chercheurs japonais, il a pu apprécier la rapidité et la douceur de l'induction anesthésique au sévoflurane chez les enfants. Le sévoflurane n'amplifie pas l'effet des catécholamines sur le coeur ; il modifie très peu la tension artérielle, entraîne moins de tachycardie réflexe que les autres anesthésiques en inhalation et il offre une émergence rapide. Plusieurs travaux semblables à celui de Crawford et coll. attestent du respect du sévoflurane pour la circulation hépatique, coronaire et cérébrale.

Même s'il n'est pas une panacée, le sévoflurane jouit de plusieurs atouts que ne partagent pas les autres anesthésiques halogénés. Le problème de sa biotransformation mérite qu'on y consacre plus de recherche mais il semble anodin dans le contexte de sûreté globale que semble offrir cet agent. Le sévoflurane pourra vraisemblablement s'établir une niche en anesthésie pédiatrique. Il ne contient pas d'atome de chlore et pourra ainsi peutêtre s'avérer moins dommageable pour la couche d'ozone que les autres anesthésiques halogénés. Le rôle éventuel que jouera cet anesthésique renaissant dans notre pratique reste à déterminer.

\section{References}

1 Wallin RF, Regan BM, Napoli MD. Sterin AJ. Sevoflurane: a new inhalational anesthetic agent. Anesth Analg 1975; 54: 758-65.

2 Holaday DA, Smith FR. Clinical characteristics and biotransformation of sevoflurane in healthy human volunteers. Anesthesiology 1981; 54: 100-6. 